\title{
Analysis of Zn I emission lines observed during a spark discharge in liquid nitrogen for Zinc nanosheet synthesis
}

\author{
T. Belmonte ${ }^{1,2, *}$, H. Kabbara ${ }^{1}$, C. Noël ${ }^{1,2}$, R. Pflieger ${ }^{3}$ \\ ${ }^{1}$ Université de Lorraine, Institut Jean Lamour, UMR CNRS 7198, NANCY, F-54042, France \\ ${ }^{2}$ CNRS, Institut Jean Lamour, UMR CNRS 7198, NANCY, F-54042, France \\ ${ }^{3}$ ICSM, Institut de Chimie Séparative de Marcoule, UMR 5257, CEA-CNRS-ENSCM- \\ Université de Montpellier, Site de Marcoule, BP17171, 30207 Bagnols sur Cèze Cedex, \\ France \\ * corresponding author. Email: thierry.belmonte@univ-lorraine.fr
}

PACS number: 52.80.Wq Discharge in liquids and solids

Keywords: Spark discharges; Submerged discharges; Time-resolved optical emission spectroscopy; Liquid nitrogen. 


\section{ABSTRACT}

High-voltage pulsed discharges of a few milliwatts in liquid nitrogen between two zinc electrodes are investigated by optical emission spectroscopy. The possibility to account by modelling for the time-evolution of selected zinc lines that are sufficiently intense gives access to the dynamics of the discharge. The intense background emission at short times (from 50 to $100 \mathrm{~ns}$ ) is satisfactorily described by Planck's continuum with a temperature of $0.9 \mathrm{eV}$ and an electron density of $10^{17} \mathrm{~cm}^{-3}$. Later on, when zinc lines start appearing in the spectrum, i.e. beyond 300 ns typically when the background emission drops, the electron density and the electron temperature decrease exponentially (from 5.3 to $3.5 \mathrm{eV}$ at $700 \mathrm{~ns}$ and from $4 \times 10^{16}$ to $10^{15} \mathrm{~cm}^{-3}$ ). The modelling of the time-evolution of three selected zinc lines at 468.01, 472.22 and $481.05 \mathrm{~nm}$ gives a coherent insight of the discharge behaviour and structure, especially in terms of species gradient and optical thickness. It is also used to check the reliability of the extracted data. 


\section{INTRODUCTION}

Spark discharges in dielectric liquids might be used to synthesize nanoparticles at high rate and at much lower cost than other physical processes like laser ablation if it was better controlled [1]. Even though breakdown is stochastic, because charge injection is not reproducible, it is possible to improve the repeatability of events by using nanosecond-pulsed discharges [2]. This approach reduces the dispersion of the observed discharge intensities and consequently, the size distribution of the synthesized nanoparticles.

Spark discharges in dielectric liquids, like discharges excited by laser or acoustic waves, are known to host complex phenomena at short times because of the extreme conditions that prevail after breakdown [3, 4]. During the first nanoseconds, the temperature is close to $1 \mathrm{eV}$ and the pressure may reach thousands of bars [5]. After several tens of nanoseconds, the pressure has exponentially decreased down to several or several tens of bar. As the gas is highly ionized, emission of continua due to electron-ion recombination, bremsstrahlung and/or black-body radiation dominates [6]. Some very intense lines are sometimes observed, in addition to these continua, like the $\mathrm{H} \alpha$ line which is commonly used to determine the electron density during inception of discharges in water, for instance. Even though pressure and temperature are high at this stage, pressure and Doppler broadenings of emission lines are known to be significantly lower than Stark broadening caused by the very high density of charged species close to emitters [7]. Erosion of metallic electrodes occurs, leading to the emission of a vapour whose excitation gives rise to broadened and shifted lines of the neutral atomic systems of metals. Line shifts and widths can be due to several mechanisms, the most important being usually due to Stark effects [8]. When the plasma cools down from tens to hundreds of nanoseconds, the lowering of the electron density reduces the importance of these effects to the expense of others, like resonance, pressure, and Doppler effects. The optical thickness also decreases, rending the medium thinner [9]. The relative importance of each broadening source will be discussed in the present work. 
The choice of the zinc system is motivated by the fact that this metal behaves uncommonly in liquid nitrogen, leading to the synthesis of nanosheets instead of more-frequently encountered nanoparticles [10] (See Fig. 1). A thorough explanation for this behaviour is still pending. The use of liquid nitrogen is practical because no nitrogen lines, either from atomic or molecular systems, ionized or not, are found in the spectrum. This greatly simplifies the analysis of the emitted light. We assume that these transitions are simply too weak to be visible or trapped in the optically thick medium.

Methods based on the so-called "six free parameters deconvolution" (SFPD) procedure were developed to determine from experimental data, parameters of given emission lines [11-15]. Here, we will determine the broadening parameters of three selected zinc lines together with plasma parameters like the electron density, the electron temperature and the pressure. Usually, high-pressure lamps are used to produce these data. In this work, such a method is applied to different zinc lines emitted by spark discharges in liquid nitrogen. Time-resolved spectra are exploited to check the reliability of extracted data, a method employed for the first time to the best of the authors' knowledge. This is not so much the parameters that are sought after in this work as the evolution of plasma parameters in media where this information is rarely available straightforwardly. Indeed, high-pressure lamps certainly provide more accurate parameters for line transitions as discussed hereinafter.

\section{EXPERIMENTAL SET-UP}

The experimental set-up was presented in detail in reference and is given as Supplemental material 1 [16]. Briefly, a pin-to-pin electrode configuration was used. Electrodes were zinc wires (1 mm in diameter - $99.9 \%$ purity). A high DC voltage power supply (Technix SR15R-1200-15 kV-80 mA) fed a solid-state switch (HTS-301-03-GSM) connected to one pinelectrode, the other electrode being grounded. The voltage rise time was $20 \mathrm{~ns}$ without ballast resistor. The applied voltage is $+10 \mathrm{kV}$ and the current reaches 60 amperes (See Supplemental material 2). The pulse duration was set at $200 \mathrm{~ns}$ typically and the plasma 
duration is about $500 \mathrm{~ns}$, as estimated from the time beyond which the current is zero (see Supplemental material 2). The power dissipated in average per pulse is between 1 and $5 \mathrm{~mW}$, typically. This average power was determined by using the method described by Timoshkin et al. [17]. The reader is referred to this work for further details.

Optical emission spectroscopy was performed with a $550 \mathrm{~mm}$ focal length monochromator (Jobin-Yvon TRIAX 550) equipped with a $100 \mathrm{gr} . \mathrm{mm}^{-1}$ grating for overall spectra in the visible range $(250-900 \mathrm{~nm})$. For the time evolution of specific lines, a $1200 \mathrm{gr} . \mathrm{mm}^{-1}$ grating was used, also in the range $(250-900 \mathrm{~nm})$. Both gratings are blazed at $500 \mathrm{~nm}$. It was coupled with a HORIBA Jobin-Yvon i-Spectrum Two iCCD detector. Each measurement is an accumulation of 50 successive spectra recorded at the same time after breakdown with an exposure time of $50 \mathrm{~ns}$. Although discharges in dielectric liquids are known to be stochastic, using a solid-state switch with a 20-ns rise time ensures a high level of reproducibility because breakdown necessarily occurs within a time window inferior to the exposure time (see reference [18] for further details). However, because of the generation of high-frequency signals by discharge current oscillations (at about $1 \mathrm{MHz}$ ), ghost lines are sometimes observed. According to S. Svanberg [19], from mechanically ruled gratings, so-called ghost lines can appear due to periodical errors in the mechanical feeding of the machine. These lines are random in time and wavelength. They are easily identified in time-resolved data, since they disappear from one spectrum to the other.

\section{THEORY}

Spark discharges in liquids are subjected to various broadening sources. As they are transient, the broadening mechanisms change in time because of the rapid evolution of the most important parameters that control the discharge's behaviour. The electron temperature, the electron density, the total pressure and the medium optical thickness are known to vary very strongly during the first instants, i.e. from 0 to $\sim 20-25 \mathrm{~ns}$ (current rise in Supplemental Material 2), more moderately from 25 to $150-200 \mathrm{~ns}$ (current drop) and only weakly beyond 
$200 \mathrm{~ns}$ (current weak oscillations). Before anything else, an optical model of the discharge is required.

\subsection{Optical model}

Any radiation emitted by a transition between an upper level and a lower level must travel through a medium which, if it is thick, can absorb and re-emit it several times. The spectral density of the radiant flux [W $\mathrm{m}^{-2} \mathrm{~Hz}^{-1} \mathrm{sr}^{-1}$ ] depends on the photon frequency $v$ and on the distance from the discharge center $r$ :

$$
d I(r, v)=(\varepsilon(r, v)-\kappa(r, v) I(r, v)) d r
$$

Absorption $k(r, v)\left[\mathrm{m}^{-1}\right]$ and emission $\varepsilon(r, v)\left[\mathrm{W} \mathrm{m}^{-3} \mathrm{~Hz}^{-1} \mathrm{sr}^{-1}\right]$ coefficients are determined from Einstein's coefficients for spontaneous emission $A_{u l}$, photo-absorption $B_{l u}$ and induced emission $B_{u l}$ :

$$
\begin{gathered}
\varepsilon(r, v)=\frac{A_{u l} n_{u}(r) h v_{0}}{4 \pi} f(r, v) \\
\kappa(r, v)=\frac{\left[B_{l u} n_{l}(r)-B_{u l} n_{u}(r)\right] h v_{0}}{c} f(r, v)
\end{gathered}
$$

where $n_{l}(r)$ et $n_{u}(r)$ are the densities of the lower and upper levels of the transition respectively. $f(r, v)$ is the normalized spectral distribution for an atomic transition at a given position $r: \int_{-\infty}^{+\infty} f(r, v) d v=1$. If a sufficiently large continuum emission $\varepsilon_{b g}(r, v)$ superimposes upon the line emission, Eq. (2) must reads: $\varepsilon(r, v)=\frac{A_{u l} n_{u}(r) h v_{0}}{4 \pi} f(r, v)+\varepsilon_{b g}(r, v)$.

The transition probability for induced emission is given by $B_{u l}=\left(c / \nu_{0}\right)^{3}\left(A_{u l} / 8 \pi h\right)$ and it is related to $B_{l u}$ by $B_{l u}=\left(g_{u} / g_{l}\right) B_{u l}$ where $g_{u}$ and $g_{l}$ are the degeneracy of the upper and lower levels. The intensity of the radiant flux is obtained after integration of Eq. (1) over the photon path: 


$$
I(v)=\frac{1}{\exp \left[-\int_{-\infty}^{+\infty} \kappa(r, v) d r\right]} \int_{-\infty}^{+\infty} \exp \left[-\int_{-\infty}^{+\infty} \kappa(r, v) d r\right] \varepsilon(r, v) d r
$$

To solve Eq.(4), the spatial density distribution must be given. Then, it must be discretized and integrated numerically [20]. A simple finite difference method is powerful enough to get accurate values for a sufficiently narrow grid. The optical thickness of the medium originates from the species gradients (see supplemental material 4). For electrons, as for neutral species, a Gaussian distribution is commonly chosen for discharges exhibiting a cylindrical symmetry $[R]$ :

$$
n_{e}(r)=N_{e}(0) \exp \left(\frac{-r^{2}}{\sigma_{e}^{2}}\right) ; n_{u}(r)=N_{u}(0) \exp \left(\frac{-r^{2}}{\sigma_{u}^{2}}\right) ; n_{l}(r)=N_{l}(0) \exp \left(\frac{-r^{2}}{\sigma_{l}^{2}}\right)
$$

Standard deviations may be specific to each specie. For the sake of simplicity, and to decrease as much a possible the number of parameters in the model, we set: $\sigma_{e}^{2}=\sigma_{u}^{2}=\sigma_{l}^{2}$. Taking a Boltzmann distribution between energy levels leads to: $\frac{N_{u}(0)}{N_{l}(0)}=\frac{g_{u}}{g_{l}} \exp \left(\frac{-h v_{0}}{k_{B} T_{e}}\right)$.

\subsection{Continuum emission}

The origin of the continuum emission took long to be partially clarified, even though this step is not required to account for the behaviour of zinc lines. However, it gives an original insight of the discharge structure, as explained hereinafter. The solution appeared with specific experiments that were run to synthesize $\mathrm{Cu} @ \mathrm{ZnO}$ core-shell nanostructures [21]. When discharges are ignited between two zinc electrodes in pure liquid nitrogen, a large and intense continuum is observed at the very beginning of the process, typically between 0 and $200 \mathrm{~ns}$ (see Supplement Material 3). However, if liquid nitrogen contains initially copper nanoparticles, the continuum is by far less intense and lines from the N II system overlap for a while, their contribution fading away after $400 \mathrm{~ns}$. The role of copper nanoparticles is unclear. But it is important to mention here that no copper lines are emitted in this case. Everything happens as if copper reduced the optical thickness of the medium without participating 
chemically. We suppose that the emission of a shockwave at the very beginning of the discharge and the subsequent formation of a bubble prevent (or strongly limit) copper nanoparticles from entering the discharge, which does not permit any electron excitation of copper. The presence of copper nanoparticles might favor the uptake of electrons by the surrounding liquid. The fact that no lines from $\mathrm{N}_{2}$ molecules and $\mathrm{N}$ non-ionized atoms are observed is assumed to be due to the optical thickness of the medium. $\mathrm{N}_{2}$ and $\mathrm{N}$ being likely much more concentrated than $\mathrm{N}^{+}$, only the lines emitted by the excited states of the ion are not trapped - we recall here that for a given optical thickness, the probability of a line to be absorbed is also linked to the concentration of the absorbing states -. As a consequence, the presence of metallic lines only in usual conditions (i.e. when no particles are introduced beforehand in the liquid) hides the fact the main contribution to the discharge is due to nitrogen, which sounds logical. Till now, the only detected ion being $\mathrm{N}^{+}$, we assume that the main ion in the early stages of the discharge ignition is this one. This leads to two conclusions: first, the energy deposited at the beginning of the discharge is sufficient to produce excited states of $\mathrm{N}^{+}$from $\mathrm{N}$, second, this production requires at least $14.53 \mathrm{eV}$ under vacuum [22] but decreases when the pressure increase thanks to the lowering of the ionization threshold. Thus, at 1000 bar, taking a mean electron temperature of $1 \mathrm{eV}$, the lowering of the ionization threshold of $\mathrm{N}$ would be around $9.74 \mathrm{eV}$, making possible ionization from $\mathrm{N}$ with electrons of $4.79 \mathrm{eV}$. We readily understand that the production of $\mathrm{N}^{+}$ions with a discharge where the electron temperature is only $1 \mathrm{eV}$ becomes possible.

The role of nitrogen is still unclear. However, one may assume from the previous results that the discharge at its very beginning is mainly composed of nitrogen rather than metal since it takes a short while (close to $100-150$ ns according to [21]) to melt the electrode and produce a burst of metallic vapor. This does not mean anyway that at the beginning there is no metal. There are other erosion mechanisms that could be at stake, like explosion of metal protrusions 
on electrodes crossed by high currents for instance. But at this stage, we can assume since $\mathrm{N}$ II lines were observed that nitrogen is the main element within the discharge.

Considering that the plasma is made of nitrogen, if we use Griem's criterion, we can estimate the relaxation time to reach equilibrium. It is a about $0.6 \mathrm{~ns}$ if we assume $\mathrm{Ne}=10^{22} \mathrm{~m}^{-3}$ and $\mathrm{Te}=11,604 \mathrm{~K}$. Therefore, we can consider the discharge at Local Thermal Equilibrium.

Among the different possible sources of continuum emission, we ruled out easily free-free and free-bound collisions because of the expected shapes of the emissivity profile versus wavelength (saw-teeth decaying profile and monotonously increasing profile - see e.g. Fig. 8 p16 in Ref. [23]). At 50 and 100 ns, as described in detail hereinafter, between the present theoretical approach of the Planck continuum and the experimental data will be obtained. In this case, the emissivity $\mathcal{E}_{P}(\nu, T)$ expressed in $\left[\mathrm{W} \cdot \mathrm{m}^{-3} \cdot \mathrm{sr}^{-1} \cdot \mathrm{Hz}^{-1}\right]$ radiated by the discharge at a given temperature $T$ is related to the Planck law by the Kirchhoff law:

$$
\varepsilon_{P}(\nu, T)=\kappa_{P}(\nu, T) B_{v}(T)
$$

with:

$$
B_{v}(T)=\frac{2 h v^{3}}{c^{2}} \frac{1}{\exp \left(\frac{h v}{k_{B} T}\right)-1}
$$

in $\left[\mathrm{W} \cdot \mathrm{m}^{-2} \cdot \mathrm{sr}^{-1} \cdot \mathrm{Hz}^{-1}\right]$. The Planck continuum is composed of a large number of individual emission lines which can be broadened and shifted. Considering only one of these lines, we can express the corresponding absorption coefficient which is linked to the cross sections for absorption and stimulated emission by:

$$
\kappa_{P}^{\mu \rightarrow l}(T)=N_{l} \sigma_{u l}-N_{u} \sigma_{l u}
$$

In terms of Einstein's coefficients, it reads:

$$
\kappa_{P}^{u \rightarrow l}(T)=\frac{h v}{c} \phi(v)\left(N_{l} B_{l u}-N_{u} B_{u l}\right)
$$


where $\phi(v) \cong 1 / \Delta v$ is the normalized shape of the spectral line (in [s]). $B_{l u}$ is the Einstein absorption coefficient and $B_{u l}$ is the stimulated emission coefficient (both terms in $\left.\left[\mathrm{m}^{3} \cdot \mathrm{J}^{-1} \cdot \mathrm{s}^{-2}\right]\right)$. If the densities are distributed according to a Boltzmann law, one finds:

$$
\kappa_{P}^{u \rightarrow l}(T)=\frac{h v}{c} \phi(v) \frac{g_{l}}{Q_{0}} B_{l u} N \exp \left(-\frac{E_{l}}{k_{B} T}\right)\left[1-\exp \left(-\frac{h v}{k_{B} T}\right)\right]
$$

as $g_{l} B_{l u}=g_{u} B_{u l} . Q_{0}$ is the partition function of the considered species of density $N$ and $g_{l}$ the statistical weight of the lower level. Values were taken from reference [24]. The estimation of $\kappa_{P}(\nu, T)$ from $\kappa_{P}^{\mu \rightarrow l}(T)$ requires a large set of basic data (energy levels of transitions, Einstein's coefficients, statistical weights, etc.) and is practically possible only a limited number of situations where all these data are available. However, thanks to databases like NIST's, it is possible to perform the complete calculation.

Once the background emission identified as a Planck continuum, which follows qualitatively an exponential decay over the visible range, the fitting procedure was applied to experimental points that followed such a behaviour, the other points being removed for a more accurate fit. At $50 \mathrm{~ns}$, there was no need to remove any point but at $100 \mathrm{~ns}$, those lying between $390 \mathrm{~nm}$ and $540 \mathrm{~nm}$ were excluded.

\subsection{Line broadening and shift sources}

Line broadening and shift sources that were taken into account in the present model are described in this section.

\subsubsection{Instrumental broadening}

The instrumental broadening was found equal to $0.08 \mathrm{~nm}$ thanks to a calibration using a HeNe laser. The line shape thus obtained was approximately Gaussian.

\subsubsection{Doppler broadening and shift}

Doppler effect induces a shift in frequency in the emitted radiance. The line profile is Gaussian and its FWHM is [25]: 


$$
\Delta v_{D}=v_{0} \sqrt{\frac{8 \ln 2 \times R T}{M c^{2}}}
$$

where $v_{0}$ is the frequency of the transition, $T$ is the gas temperature and $M$ is the molar weight (expressed in $\left.\left[\mathrm{kg} \cdot \mathrm{mol}^{-1}\right]\right)$ of radiating atoms.

The line shift due to the Doppler effect is:

$$
d_{D}=\frac{v}{c} v_{0}
$$

where $v$ is the atom velocity.

\subsubsection{Pressure broadening and shift (impact approximation)}

The following van der Waals broadening expressions are valid only within the impact approximation.

The shift in frequency is given by the following expression:

$$
\Delta v_{v d w}=\left|C_{6}^{\prime}\right|^{2 / 5} \bar{v}^{3 / 5} N
$$

where $C_{6}^{\prime}\left[m^{6} \cdot s^{-1}\right]=\frac{C_{6}\left[J \cdot m^{6}\right]}{\hbar} . C_{6}$ is the van der Waals coefficient. It can be estimated from the Lennard-Jones coefficients $\left(\varepsilon_{0}, \sigma\right)$ knowing that $C_{6}=4 \varepsilon_{0} \sigma^{6}$. We used the data published by Born [26].

Lines purely broadened by van der Waals interactions possess a $T^{0.3}$ dependence for $\Delta v_{v d w}$ and $d_{v d w}$ (with the same unit as $\Delta v_{v d w}$ ), as predicted by Foley and Lindholm [27] who also showed that:

$$
d_{v d w} / \Delta v_{v d w}=-0.363
$$

The line is usually red-shifted (minus sign). The line shape is Lorentzian.

We used the criterion of Hindmarsh [28] based on the calculation of the Weisskopf radius and its comparison with the mean distance between pertubers to define the validity of the impact approximation (see [23] for details).

\subsubsection{Pressure broadening and shift (quasi-static approximation)}


In the quasi-static approximation, the FWHM expressed in frequency is given by:

$$
\Delta v_{v d w}^{q s}=\frac{1}{2 \pi}\left|\hat{C}_{6}^{\prime}\left(\frac{4 \pi}{3} N \Gamma\left(\frac{1}{2}\right) \cos \left(\frac{3 \pi}{12}\right)\right)^{2}=\frac{4 \pi^{2}}{9}\right| \hat{C}_{6}^{\prime} \mid N^{2}
$$

Here, $\hat{C}_{6}^{\prime}$ can be slightly different from $C_{6}^{\prime}$, the difference could be associated with dependences on the angular momentum quantum numbers [29]. The line profile is asymmetric and its form is taken according to Margenau [30]:

$$
M(v)=\left\{\begin{array}{cc}
-\sqrt{\frac{\Delta v_{v d w}^{q s}}{\left(v-v_{0}\right)^{3}}} \exp \left(-\frac{\pi}{4} \frac{\Delta v_{v d w}^{q s}}{v-v_{0}}\right) & \text { if } v<v_{0} \\
0 & \text { if } v \geq v_{0}
\end{array}\right.
$$

For line shift, one may refer to reference [23].

\subsubsection{Resonance broadening}

It occurs for transitions involving a level that is dipole-coupled to the ground state (i.e. the upper or lower level having an electric dipole transition and the corresponding force derives from the potential $\Delta V=\hbar C_{3} / r^{3}$ ). Because of this, the following transitions must be considered: $g \rightarrow l ; g \rightarrow u$ and $l \rightarrow u$ where $g, l$ and $u$ are the ground, upper and lower levels. When present, the resonance broadening is usually the dominant effect and at low densities the shape is Lorentzian. The FWHM is given by:

$$
\Delta v_{r e s}=\pi k_{g l} C_{3, g l}^{\prime} \sqrt{\frac{g_{g}}{g_{l}}} N_{g}
$$

$k_{g l}$ is a numerical constant for the transition between the lower level and the ground level.

We used $k_{g l}=1.53[31]$.

Resonance broadening usually results in unshifted dispersion Lorentzian profiles [32].

\subsubsection{Stark broadening}

The Stark profiles of atomic lines in plasma are produced predominantly under the action of high-frequency fields of electrons (the linear Stark effect, which causes a shift in energy

which is linear in the field strength with $V_{2}=-C_{2} / r^{2}$ ) and under the action of the low- 
frequency fields of ions (the quadratic Stark effect, which causes a shift in energy which is quadratic in the field strength $\left.V_{4}=-C_{4} / r^{4}\right)$. In the case of non-hydrogenoid atomic transition, ion broadening is not negligible and line profiles become asymmetric. If the quasi-static approximation applies, the line profile is given by:

$$
j_{A, R}(x)=\frac{1}{\pi} \int_{0}^{\infty} \frac{H_{R}(\beta)}{1+\left(x-A^{4 / 3} \beta^{2}\right)^{2}} d \beta
$$

where $x=\frac{\lambda-\lambda_{0}-d_{e}}{w_{e}}, w_{e}$ is the HWHM of the Stark broadening due to electron collision and $d_{e}$ is the corresponding shift. $H_{R}(\beta)$ is the distribution function of the micro-field intensity that depends on the normalized intensity of the Holtsmark field $\beta=F / F_{0}, F_{0}$ being the intensity of the Holtsmark field. $A$ is the broadening parameter due to static ions. $R$ is the ratio of the average distance between ions and the Debye radius, i.e. the screening parameter of Debye:

$$
R=\left[\frac{36 \pi e^{6} N_{e}}{\left(k_{B} T_{e}\right)^{3}}\right]^{1 / 6} \approx 0.0899 \frac{\left(N_{e}\left[\mathrm{~cm}^{-3}\right]\right)^{1 / 6}}{T_{e}^{1 / 2}}
$$

The corresponding profiles have been calculated by Hooper [33]. For the limit case of Holtsmark, i.e. for $R=0$, the shape of the function is given by:

$$
H_{R=0}(\beta)=H_{0} \beta \int_{0}^{\infty} x \sin (\beta x) \exp \left(-x^{3 / 2}\right) d x
$$

where $H_{0}$ is a normalization constante.

When the ion dynamic is no longer negligible, it is necessary to introduce a correction factor. The criterion to fulfill is then [11]:

$$
B=A^{1 / 3} \frac{8.06 \times 10^{-7} w_{e}^{r e f}[\mathrm{~nm}]}{(\lambda[\mathrm{nm}])^{2}}\left(N_{e}\left[m^{-3}\right]\right)^{2 / 3} \sqrt{\frac{\mu[\mathrm{amu}]}{T_{g}[\mathrm{~K}]}}<1
$$

where $w_{e}^{\text {ref }}$ is the value of $w_{e}$ when $N_{e}=10^{23} \mathrm{~m}^{-3} \cdot \mu$ is the reduced mass of the ion or neutral perturber in arbitrary mass unit. This means that strong collisions due to electrons and ions are well separated in time. Then, the total broadening is given by:

$$
\Delta \lambda_{s}=2 w_{e}\left[1+1.75 A D_{J}(1-0.75 \kappa R)\right]
$$


with $D_{J}=\frac{1.36}{1.75(1-0.75 R)} B^{-1 / 3}$ if $B<\left(\frac{1.36}{1.75(1-0.75 R)}\right)^{3}$ or $D_{J}=1$ if $B \geq\left(\frac{1.36}{1.75(1-0.75 R)}\right)^{3}$

[11]. In this latter case, ion dynamic is negligeable and the line shape is treated by considering the quasi-static approximation.

Approximate formulas, resorting to parametric functions, were derived for an easier use. They are reasonably accurate if $0.05 \leq \alpha \leq 0.5$ and $r \leq 0.8$, these two quantities being defined hereafter. The line width broadened by Stark effect is then given by:

$$
\Delta \lambda_{s}=2\left[1+1.75 \alpha\left(1-0.75 \kappa r_{D}\right)\right] w
$$

where $w\left(T_{e}, N_{e}\right)$ and $\alpha\left(T_{e}, N_{e}\right)$ are two parametric functions tabulated by [32]. They represent the electronic contribution expressed in wavelength and the ion quasi-static broadening parameter. They scale respectively as $N_{e}$ and $N_{e}^{1 / 4}$. So, they are more conveniently written as $w\left(T_{e}, N_{e}\right)=\frac{N_{e}}{N_{e}^{0}} w_{e}\left(T_{e}\right)$ and $\alpha\left(T_{e}, N_{e}\right)=\left(\frac{N_{e}}{N_{e}^{0}}\right)^{1 / 4} a\left(T_{e}\right)$ where $w_{e}$ and $a$ are dependent on $T_{e}$ only. $N_{e}$ is the electron density and $N_{e}^{0}=10^{22} \mathrm{~m}^{-3} \cdot r_{D}=\rho_{m} / \rho_{D}$ is the Debye shielding parameter. It is the ratio of the mean distance between ions $\rho_{m}=\left(\frac{4}{3} \pi N_{e}\right)^{-1 / 3}$ and the Debye radius $\rho_{D}=\sqrt{\frac{\varepsilon_{0} k_{B} T_{e}}{e^{2} N_{e}}} \cdot r_{D} \approx 8.899 \times 10^{-3} N_{e}^{1 / 6} T_{e}^{-1 / 2} . \kappa$ is a constant equal to 1 for a neutral emitter and 1.6 for an ion emitter. Equation (22) reads:

$$
\Delta \lambda_{s}=2 \times 10^{-22}\left[1+5.534 \times 10^{-6} N_{e}^{1 / 4} a\left(T_{e}\right)\left(1-6.742 \times 10^{-3} \kappa N_{e}^{1 / 6} T_{e}^{-1 / 2}\right)\right] N_{e} w_{e}\left(T_{e}\right)
$$

or, expressed in frequency (in $[\mathrm{Hz}])$ :

$$
\Delta v_{s}=2 \times 10^{-22} \frac{v_{0}^{2}}{c}\left[1+5.534 \times 10^{-6} N_{e}^{1 / 4} a\left(T_{e}\right)\left(1-6.742 \times 10^{-3} \kappa N_{e}^{1 / 6} T_{e}^{-1 / 2}\right)\right] N_{e} w_{e}\left(T_{e}\right)
$$

Here, $v_{0}$ is the central frequency of the unperturbed line.

The line shift is given by:

$$
d_{s}=d \pm 2 d\left(1-0.75 \kappa r_{D}\right) w
$$


$d\left(T_{e}, N_{e}\right)$ is a parametric function tabulated by Griem [32]. It represents the electron impact shift (expressed in m). It scales as $N_{e}$ and it is usually written as $d\left(T_{e}, N_{e}\right)=\frac{N_{e}}{N_{e}^{0}} d_{e}\left(T_{e}\right)$.

$$
d_{s}=1 \times 10^{-22}\left[\frac{d_{e}}{w_{e}} \pm 6.325 \times 10^{-6} N_{e}^{1 / 4} a\left(T_{e}\right)\left(1-6.742 \times 10^{-3} \kappa N_{e}^{1 / 6} T_{e}^{-1 / 2}\right)\right] N_{e} w_{e}\left(T_{e}\right)
$$

The sign \pm is equal to that of the low-velocity limit for $d_{e}$. As line profiles are asymmetric, the shift at half width is different from the line shift and it can be determined by multiplying the term $6.325 \times 10^{-6}$ by 1.6 [25]. Griem's tables (Table 4.5 p 454 and Table 4.6 p 507 in [23]) give $w_{e}$ (in $[\AA]$ ), $a$ and $d_{e} / w_{e}$ between $T_{e}=2,500$ and $T_{e}=80,000 \mathrm{~K}$ for visible and UV lines of neutral and singly ionized atoms from helium to calcium. Several reviews of experimental Stark widths and shifts for spectral lines of neutral and ionized atoms are also available [34] and [35] and can be used.

If, on the other hand, $D_{J}>1$, this parameter must be included in the calculation. Then, the method developed by Barnard et al. [36] must be used to determine the profile shape. This situation was not encountered in our work $\left(D_{J}=1\right)$.

The expression of $j_{A, R}(x)$ is determined thanks to the Woltz tables [37] from $w_{e}$, the profile being determined with $\Delta \lambda_{s}$.

\section{RESULTS AND DISCUSSION}

Experimental results giving the time-evolution of discharge emissions are presented in Fig. 2a. These data were recorded with a low-resolution grating $\left(100 \mathrm{gr} . \mathrm{mm}^{-1}\right)$. From then on, it is possible to interpret the background emission thanks to the method described previously. Changes of the background emission between 50 and 200 ns are displayed in Fig. 3a. By using a Lorentz profile for N II each line referenced in the data and setting a FWHM equal to $10 \mathrm{~nm}$, i.e. large enough to get a continuum - which suppress the possibility to estimate the 
pressure -, we could fit very accurately the background emission (Fig. 3b). The fact that we have not access to an absolute value of the emissivity, but only to shape of the distribution suppresses the possibility to estimate the electron density. The theoretical results obtained for the behaviours shown in Fig. 3b were determined by setting the electron density at $10^{17} \mathrm{~cm}^{-3}$, a value that is coherent with other results presented later. Then, the electron temperature was found to decrease from 0.9 to $0.7 \mathrm{eV}$ from 50 to $100 \mathrm{~ns}$.

Even though this approach is yet to be confirmed and is not essential for the reasoning exposed hereafter, it gives for the first time a new vision of what this specific type of discharges can be just after ignition. It is made of a medium where most nitrogen ionic lines are thick and probably much more intense than metallic lines that are hidden in the continuum. We know from studies made with picosecond-pulsed discharges [38] that breakdown in nanosecond-pulsed discharges are composed of both thermal and electric stress components, the second one being responsible for the lowering of the liquid density before the electrode tip. We also know from other works [39], that the time required to melt most metal (in fact those with melting temperature higher than that of aluminium) when submitted to a discharge in our condition is about 100-150 ns. So, the burst of the metallic vapour coming from the electrode erosion occurs relatively late. At the very beginning, the presence of metal in the discharge might be due to other phenomena, like explosion of surface protrusion, for instance. With such a behaviour, we readily understand that the primary discharge may not be composed predominantly of the metallic vapour but actually of a large part of nitrogen. This shed light on the essential need for an accurate diagnostics of the first stages of the discharge.

Besides the intense background emission that lasts approximatively during the first $300 \mathrm{~ns}, 2$ main groups of zinc emission lines found around $330 \mathrm{~nm}$ and $470 \mathrm{~nm}$ and an isolated line at $636.23 \mathrm{~nm}$ are observed (see Table 1 for a list of all observed transitions). The Grotrian diagram of zinc, where transitions corresponding to emitted lines are depicted, is given as 
supplemental material (see supplemental material 5). Raw data must be corrected from the optical response of the device to get exploitable results (Fig. 2b). Once done, this operation gives access to prime information about line shift and shape. The best set of results was obtained with a high-resolution grating $\left(1200 \mathrm{gr} . \mathrm{mm}^{-1}\right)$ for lines around $470 \mathrm{~nm}$. Other systems are noisier and could not be exploited by lack of accuracy (see supplemental material 6). Changes of lines shape and position are clearly observed in Fig. 4. The three zinc transitions observed between 465 and $485 \mathrm{~nm}$ exhibit a non-symmetric shape and an important red shift. The time-evolution of the line position and the corresponding shift determined from experimental data is given in Figs. 5a and $\mathbf{5 b}$ respectively.

Both features of transition lines (shape and shift) could be reproduced very accurately thanks to our model. This is shown in Fig. 6. We kept as many parameters as possible constant in the model, to avoid overparameterization of the problem. The number of degrees of freedom was then as small as possible to get a reliable and robust solution. Pressure was set at 8 bar, a realistic value at this stage of the discharge evolution [7]. From the set of data available from 300 to $700 \mathrm{~ns}$, we could determine values of the basic parameters required in Eq. (27) - ( $w_{e}$, $d_{e}$ and $a\left(T_{e}\right)$ ), i.e. 6 parameters for the 3 zinc lines - to account for the stark broadening (see Table 2). This is the main contribution at short times (300 s), but not at 700 ns where other broadening sources have non-negligible contribution (Table 3). To the best of our knowledge, these values have never been estimated before for the selected zinc transitions. To sum up, $w_{e}$, $d_{e}$ and $a\left(T_{e}\right)$ from Eq. 27 for the 3 selected transition, $N_{e}, T_{e}, \sigma_{\mathrm{e}}$ from Eq. (5) were taken as parameters.

As mentioned in the introduction, lamps are probably more suited to get accurate values for these parameters since the present method is applied to a stochastic system, discharges being not strictly reproducible from one event to another.

The evolution of $\sigma_{e}$ in Eq. (5), which is related to the optical thickness, varies typically of 0.003 to $0.0008 \mathrm{~m}$ during the investigated time range. The optical thickness of the discharge 
decreases as expected. Typically, we have found that the optical thickness varies from $2.36 \%$ (resp. $4.34 \%$ and $7.08 \%$ ) to $0.030 \%$ (resp. $0.18 \%$ and $0.27 \%$ ) for line at 468 (resp. 472 and $481 \mathrm{~nm})$.

The time evolutions of electron temperature and electron density (the last two variables of our model among the 12 considered) are depicted in Fig. 7. Both the electron temperature and the electron density behave alike versus time. Extrapolation of these behaviours at short times indicates that the electron density should be around several $10^{17} \mathrm{~cm}^{-3}$ whereas the electron temperature should be slightly below $0.8 \mathrm{eV}$. These values do not account for the pressure rise that certainly occurs in these conditions. So, setting $N_{e}=10^{17} \mathrm{~cm}^{-3}$ and $T_{e}=0.9 \mathrm{eV}$ to reproduce the background emission after $50 \mathrm{~ns}$ as we did is actually coherent with the information obtained from the modelling of the zinc lines evolution.

\section{CONCLUSION}

Describing accurately the time-evolution of a discharge in liquid nitrogen is a powerful approach to account for the evolution of the medium and of its structure. We have developed a complete model taking into account the optical thickness of the plasma, the background emission sources, the different mechanisms responsible for the broadening and shift of the emission lines observed during time-resolved measurements performed by optical emission spectroscopy in the visible range. In the present case, we could determine the time evolution of some plasma parameters like the electron density (between $10^{15}$ and $5 \times 10^{16} \mathrm{~cm}^{-3}$ between 300 and $700 \mathrm{~ns}$ after breakdown) and the electron temperature (between 0.3 and $0.5 \mathrm{eV}$ in the same time range) for a mean pressure around 8 bars. Some values of basic parameters required for stark broadening of three selected zinc lines (transitions at 468.01, 472.22 and $481.05 \mathrm{~nm}$ ) could also be determined: $w_{e}, d_{e}$ and $a$ appearing in Eq. (27). This might open ways to better understand how electrode erosion and the subsequent emission and condensation of a metallic vapour give rise to particles synthesis. Especially in the case of zinc, it turns out that zinc condensates under a two-dimensional shape, which is unusual in 
this kind of process. Accessing to the onion-structure of the optically-thick discharge could explain how the gradient density could favour or not the synthesis of $2 \mathrm{D}$ objects at layer boundaries. 


\section{REFERENCES}

[1] Belmonte T, Hamdan A, Kosior F, Noël C, Henrion G (2014) J. Phys. D: Appl. Phys. 47 224016

[2] Seepersad Y, Pekker M, Shneider M N, Fridman A, Dobrynin D 2013 J. Phys. D: Appl. Phys. 46355201

[3] Vogel A, Busch S, Parlitz U 1996 J. Acoust. Soc. Amer. 100148

[4] Pflieger R, Brau H P, Nikitenko S I 2010 Chem.-A Europ. J. 1611801

[5] Marinov I, Starikovskaia S, Rousseau A 2014 J. Phys. D: Appl. Phys. 47224017

[6] Descoeudres A, Hollenstein C, Demellayer R, Wälder G 2004 J. Phys. D: Appl. Phys. 37 875

[7] Hamdan A, Marinov I, Rousseau A, Belmonte T 2014 J. Phys. D: Appl. Phys. 47055203

[8] Tortai J H, Bonifaci N, Denat A, Trassy C 2005 J. Appl. Phys. 97053304

[9] Wendt M, Franke S 2008 J. Phys. D: Appl. Phys. 41144018

[10] Kabbara H, Ghanbaja J, Noël C, Belmonte T 2018 Mater. Chem. Phys. 207350

[11] Milosavljević V, Poparić G 2001 Phys. Rev. E 63036404

[12] Nikolić D, Djurović S, Mijatović Z, Kobilarov R 2003 Phys. Rev. E 67058401

[13] Nikolić D, Djurović S, Mijatović Z, Kobilarov R, Konjević N 2001 J. Appl. Spectrosc. 68902

[14] Milosavljević V, Ellingboe A R, Djeniže S 2006 Spectrochim. Acta B 6181

[15] Ivković M, Zikic R, Jovićević S, Konjević N 2006 J. Phys. B: At. Mol. Opt. Phys. 39 1773

[16] Hamdan A, Noel C, Kosior F, Henrion G, Belmonte T 2013 J. Appl. Phys. 113043301

[17] Timoshkin I V, Fouracre R A, Given M J, MacGregor S J 2006 J. Phys. D: Appl. Phys. 394808 
[18] Hamdan A, Noël C, Ghanbaja J, Belmonte T 2014 Plasma Chem. Plasma Process. 34 1101

[19] Svanberg S 2012 Atomic and Molecular Spectroscopy: Basic Aspects and Practical Applications (Springer Science \& Business Media) pp. 105

[20] Sakka T, Nakajima T, Ogata Y H 2002 J. Appl. Phys. 922296

[21] Kabbara H, Ghanbaja J, Noël C, Belmonte T 2017 Nano-Structures Nano-Objects 1022

[22] Kramida A, Ralchenko Yu, Reader J, NIST ASD Team (2018) NIST Atomic Spectra Database (ver. 5.5.6), [Online]. Available: https://physics.nist.gov/asd [2018, May 5]. National Institute of Standards and Technology, Gaithersburg, MD

[23] Belmonte T, Noël C, Gries T, Martin J, Henrion G 2015 Plasma Sources Sci. Technol. 24 064003

[24] Irwin A W 1981 Astrophys. J. Suppl. Series 45621

[25] Konjević N 1999 Phys. Reports 316339

[26] Born M 1999 J. Phys. D: Appl. Phys. 322492

[227] Lindholm E 1941 Ark. Mat. Astron. Och. Fys. 28B ; Foley H M 1946 Phys. Rev. 69616

[S] Hindmarsh W R, Du Plessis A N, Farr J M 1970 J. Phys. B: At. Mol. Opt. Phys. 3 L5

[29] Demura A V, Umanskii S Ya, Scherbinin A V, Zaitsevskii A V, Demchenko G V, Astapenko V A, Potapkin B V 2011 Int. Rev. At. Mol. Phys. 2109

[30] Margenau H 1935 Phys. Rev. 48755

[31] Malvern A R, Pinder A C, Stacey D N, Thompson R C 1980 Proc. Royal Soc. London A 371259

[32] Griem H R 1964 Plasma spectroscopy (New York: Mc Graw-Hill)

[33] Hooper C F 1966 Phys. Rev. 14977

[34] Konjević N, Roberts J R 1976 J. Phys. Chem. Ref. Data 5209

[35] Konjević N, Lesage A, Fuhr J R, Wiese W L 2002 J. Phys. Chem. Ref. Data 31819 
[36] Barnard J, Cooper J, Smith E W 1974 J. Quant. Spectrosc. Radiat. Transf. 141025

[37] Woltz L A 1986 J. Quant. Spectrosc. Radiat. Transfer 36547

[38] Starikovskiy A, Yang Y, Cho Y I, Fridman A 2011 Plasma Sources Sci. Technol. 20 024003

[39] Hamdan A, Kosior F, Noel C, Henrion G, Audinot J-N, Gries T, Belmonte T 2013 J. Appl. Phys. 113213303 


\section{CAPTIONS}

Figure 1: Image by transmission electron microscopy of two-dimensional zinc oxide nanosheets after synthesis in liquid nitrogen and air exposure. Synthesis conditions: pure zinc electrodes. Gap distance: $100 \mu \mathrm{m}$. Applied voltage: $10 \mathrm{kV}$ during $200 \mathrm{~ns}$.

Figure 2: Time-evolution of the discharge emission a) without and b) with correction of the instrument spectral response. Grating: 100 gr. $\mathrm{mm}^{-1}$.

Figure 3: a) Time-evolution of the first spectra recorded between 50 and $200 \mathrm{~ns}$ and b) Theoretical Planck's continuum used to fit the two first experimental data.

Figure 4: Time-evolution of the three zinc lines observed between 465 and $485 \mathrm{~nm}$ between 250 and $650 \mathrm{~ns}$.

Figure 5: Time-evolution of the shifts of the selected lines.

Figure 6: a) Comparison of theoretical spectra and experimental data for the selected zinc lines.

Figure 7: Time-evolution of the electron temperature and electron density determined theoretically.

Table 1: Basic data used in the model and taken from reference [19].

Table 2: Parameters of Eq. (27) as determined from experimental data for the three studied zinc lines.

Table 3: Contribution of broadening sources expressed in \% at two different times.

Supplemental Material 1: Schematic of the experimental setup used in this work.

Supplemental Material 2: Voltage and current time-evolutions. Discharge in liquid nitrogen between two zinc electrodes in pin-to-pin configuration. Applied voltage: $+10 \mathrm{kV}$. 
Supplemental Material 3: Intensity emission $100 \mathrm{~ns}$ after breakdown. Discharge between zinc electrodes in liquid nitrogen with or without copper nanoparticles. Besides N II lines, only $\mathrm{Zn}$ I lines are detected in the time-resolved spectra in both situations (no copper). These data are not corrected from the optical response of the system used to record them.

Supplemental Material 4: The optical model used in this work

Supplemental Material 5: Grotrian diagram of zinc showing the main transitions observed in this work.

Supplemental Material 6: Example of time-evolution of weak lines. These lines are too noisy to be exploited. 


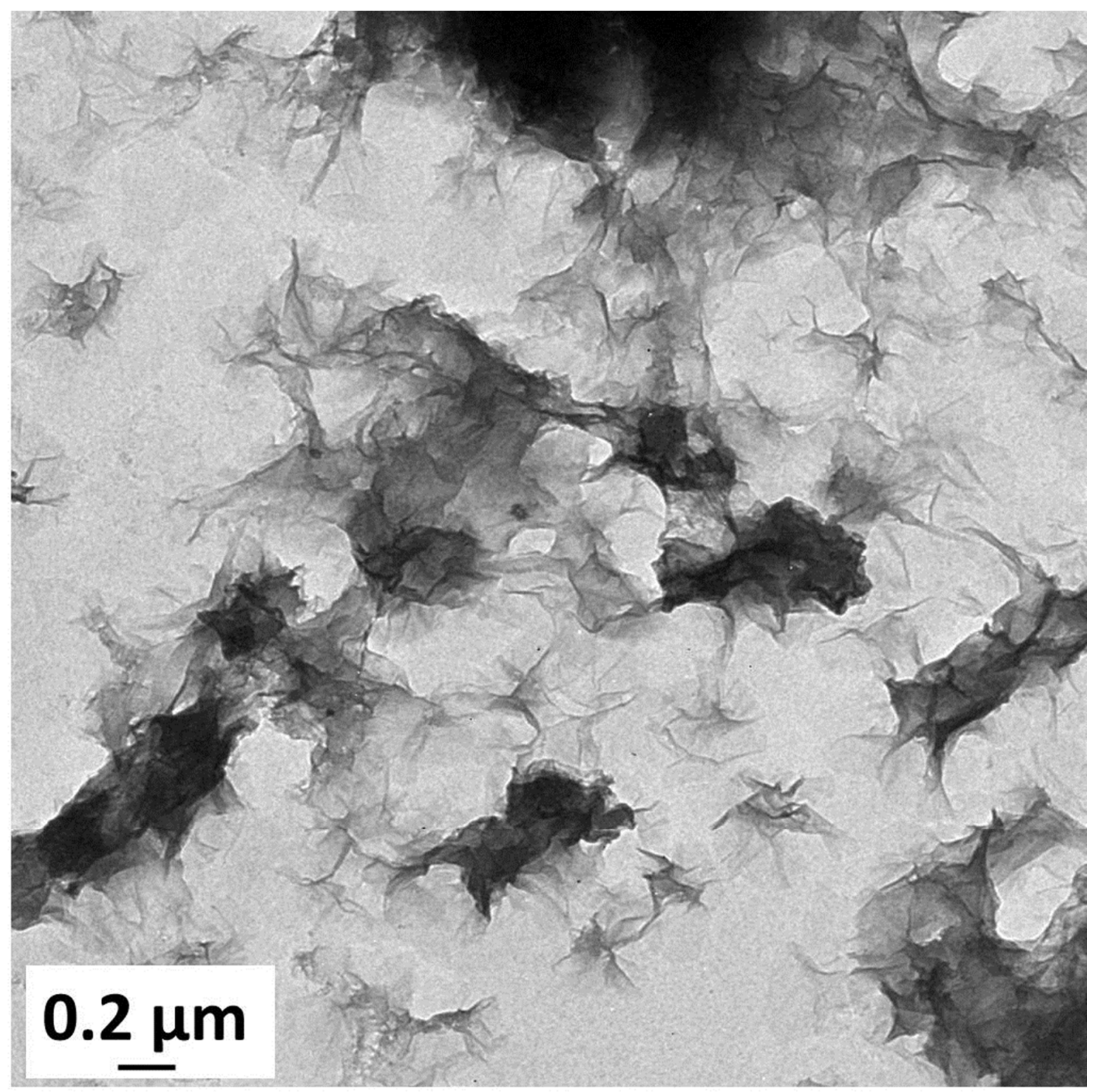

Figure 1: Two-dimensional zinc oxide nanosheets after synthesis in liquid nitrogen and air exposure. 

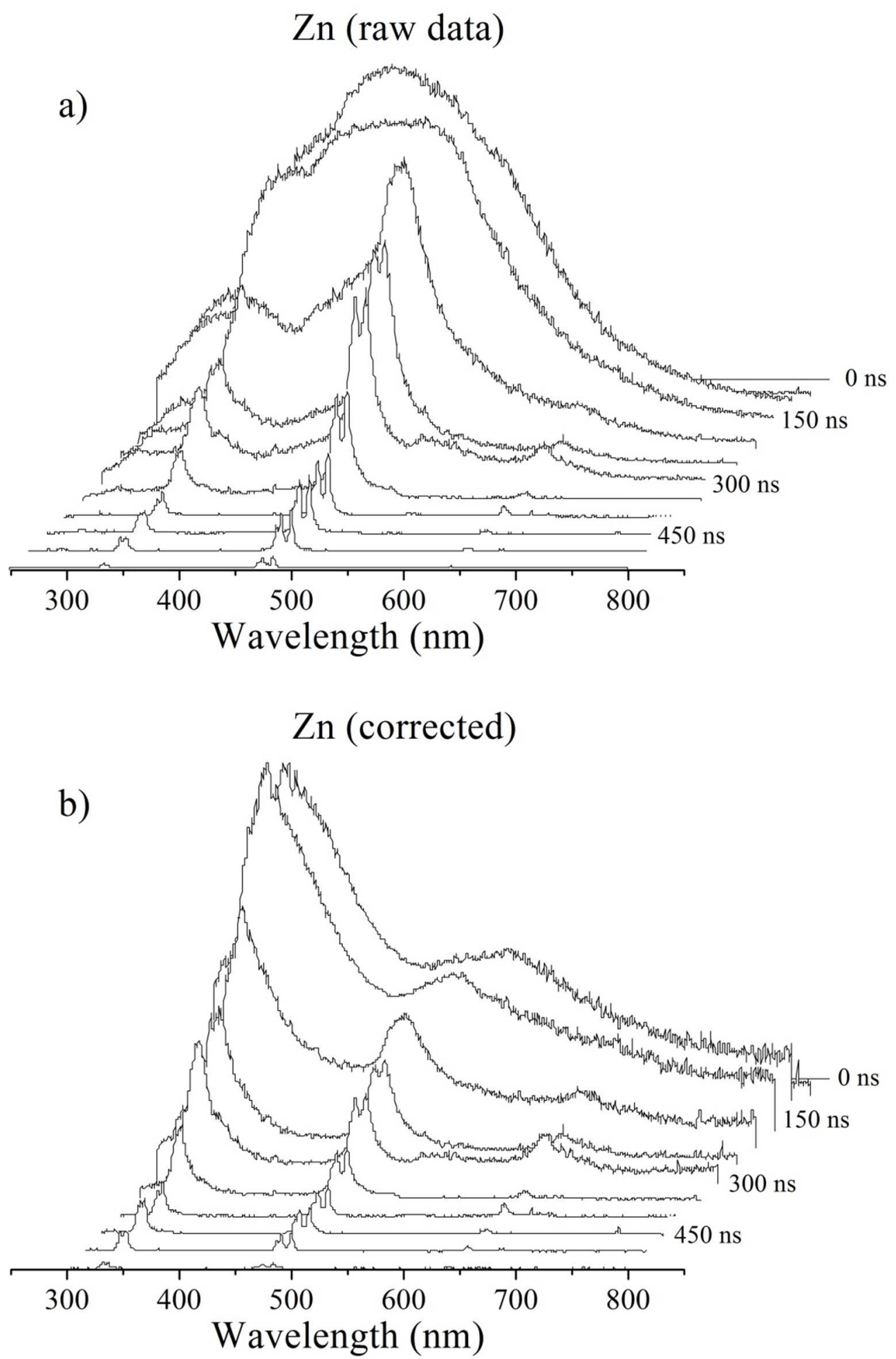

Figure 2: Time-evolution of the discharge emission a) without and b) with correction of the instrument spectral response. Grating: $100 \mathrm{gr} . \mathrm{mm}^{-1}$. 

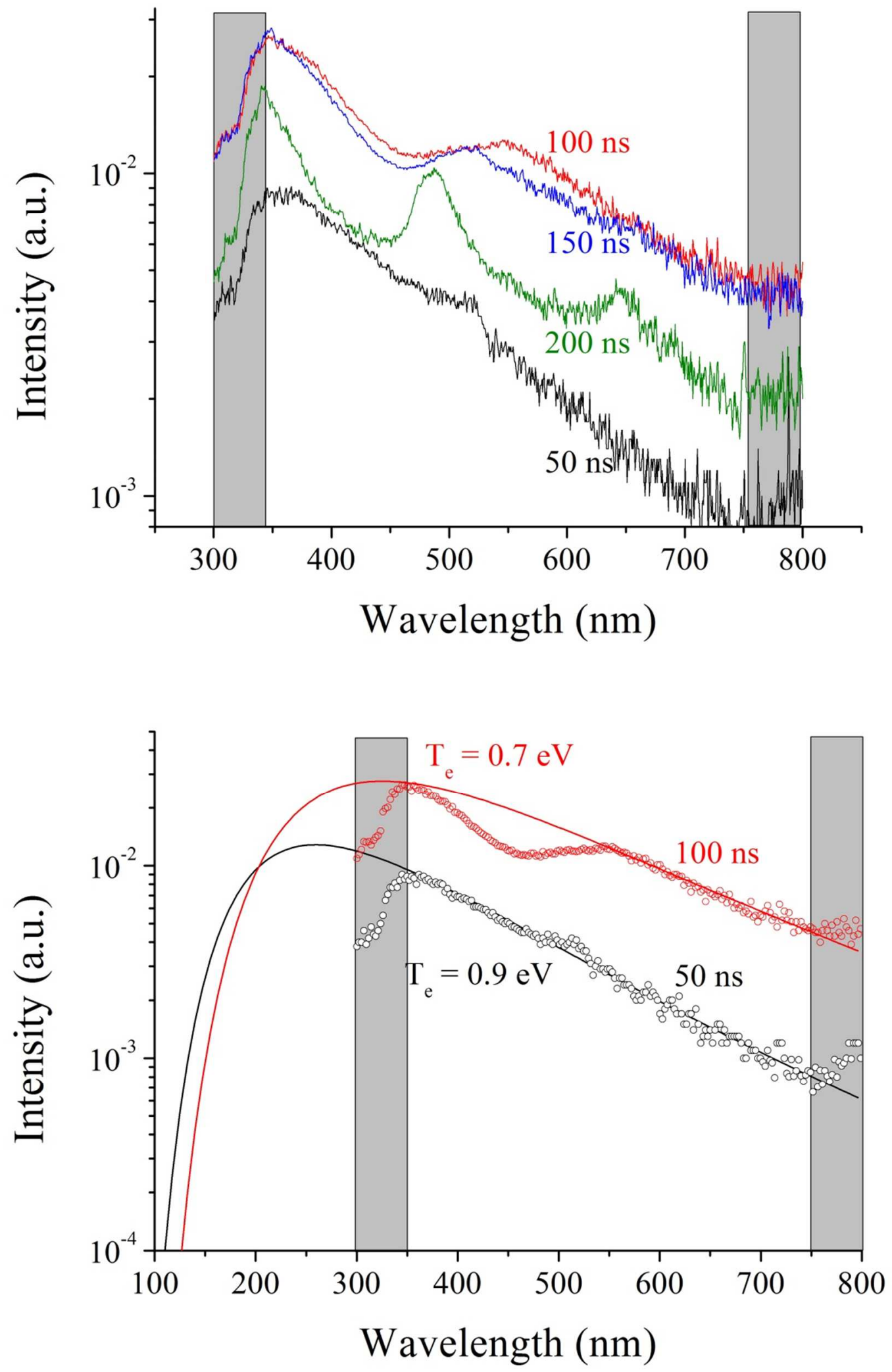

Figure 3: a) Time-evolution of the first spectra recorded between 50 and $200 \mathrm{~ns}$ and b) Theoretical Planck's continuum used to fit the two first experimental data. 


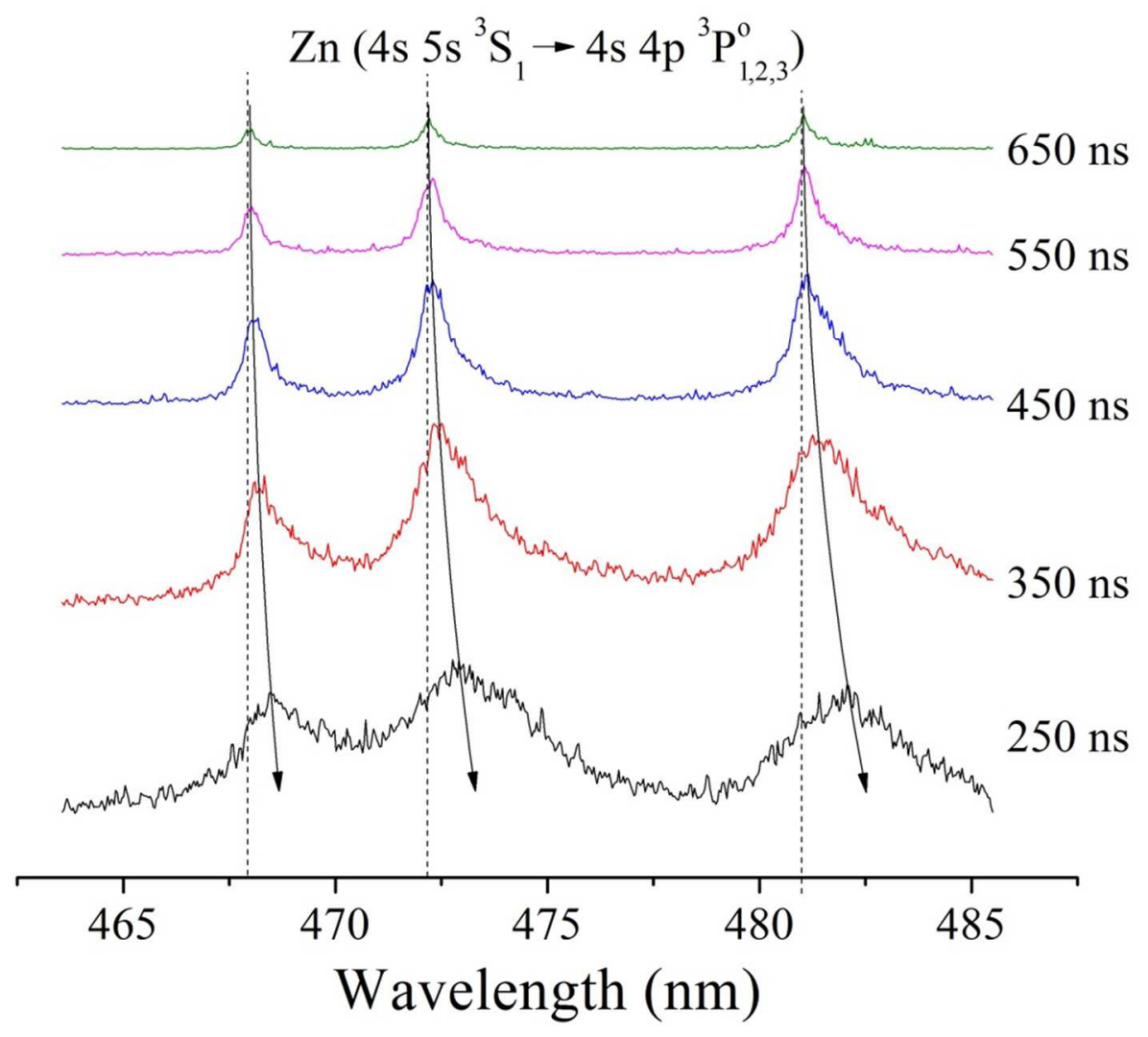

Figure 4: Time-evolution of the three zinc lines observed between 465 and $485 \mathrm{~nm}$ between 250 and $650 \mathrm{~ns}$. 


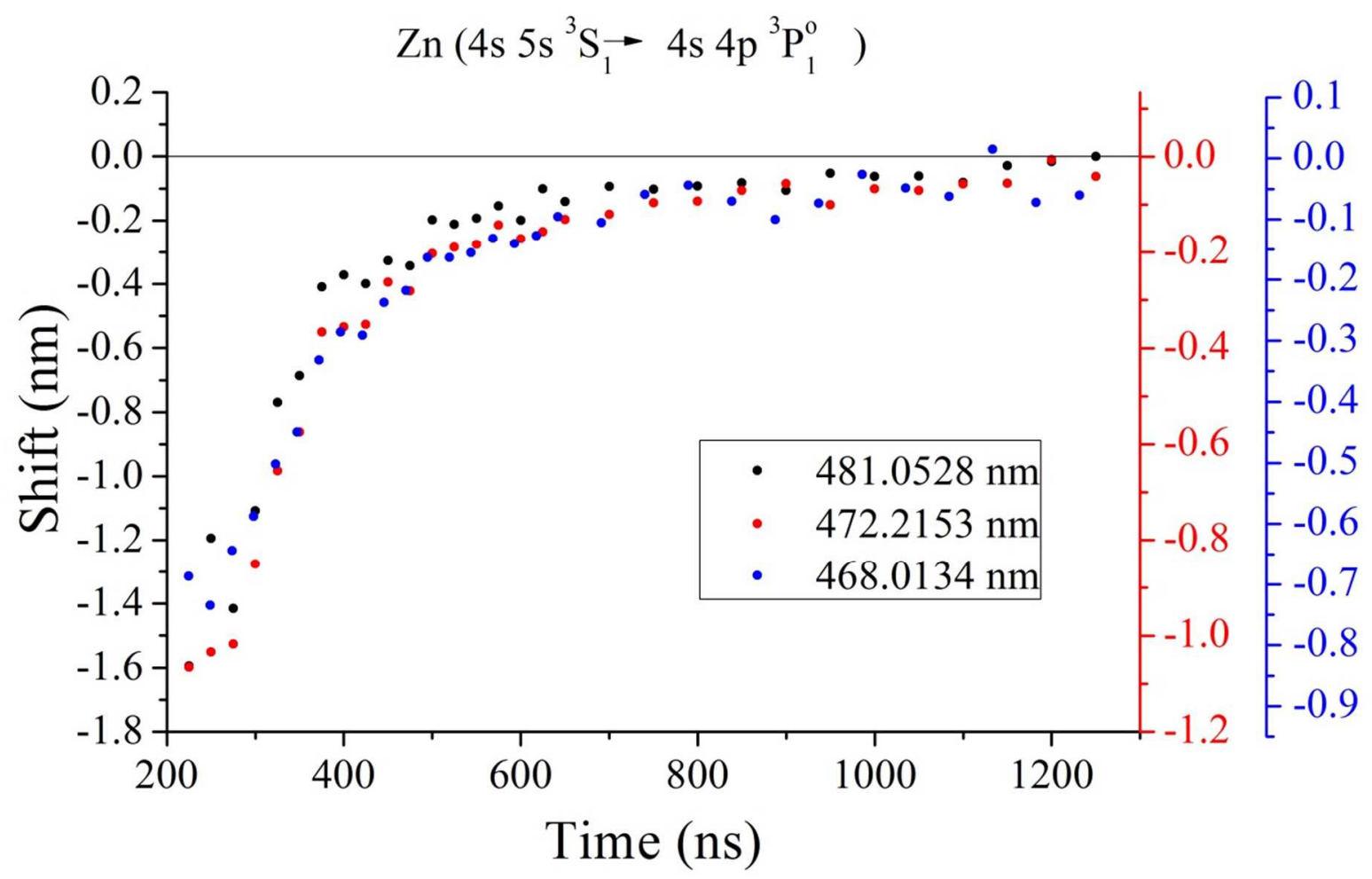

Figure 5: Time-evolution of the shifts of the selected lines. 


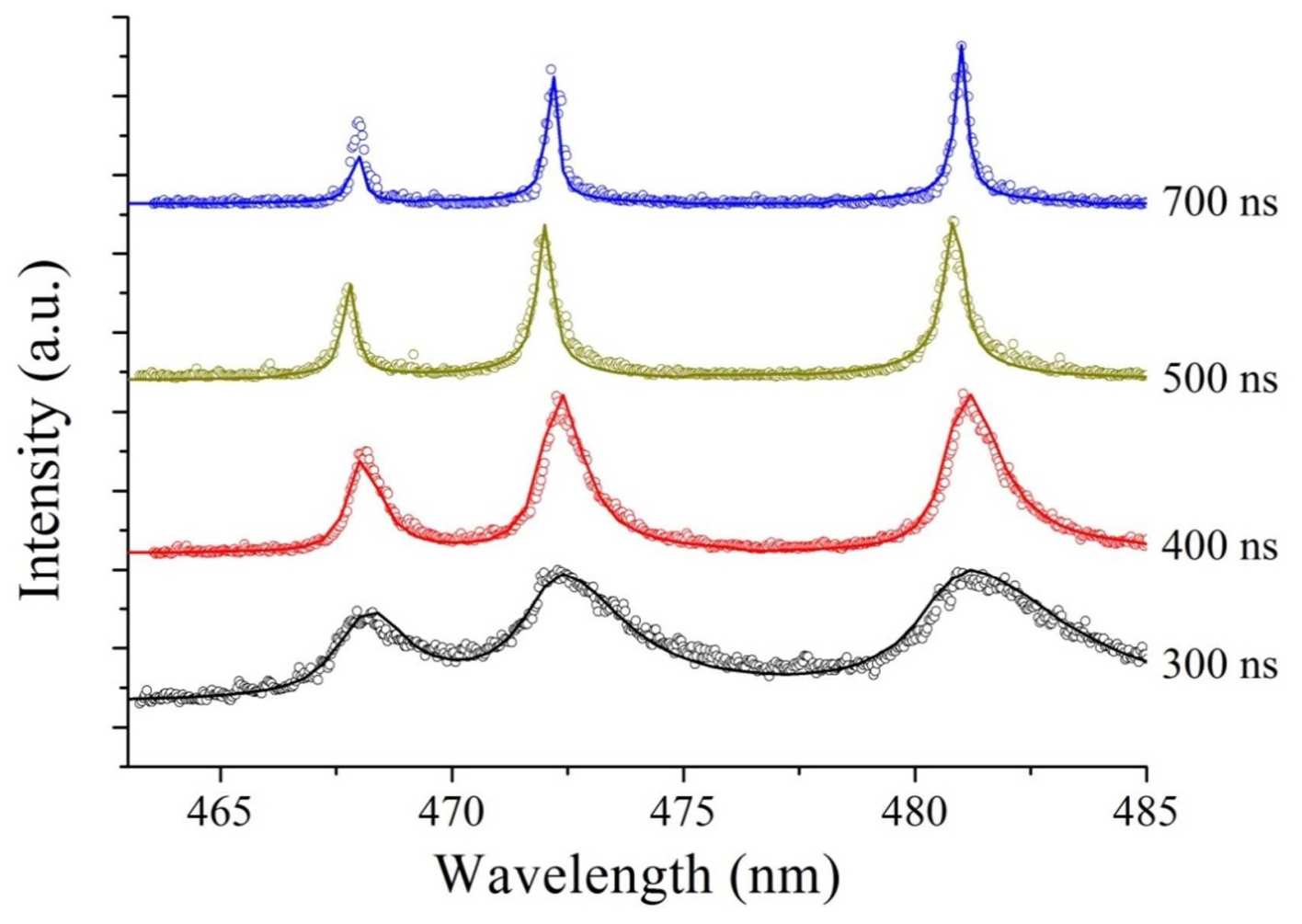

Figure 6: a) Comparison of theoretical spectra and experimental data for the selected zinc lines. 


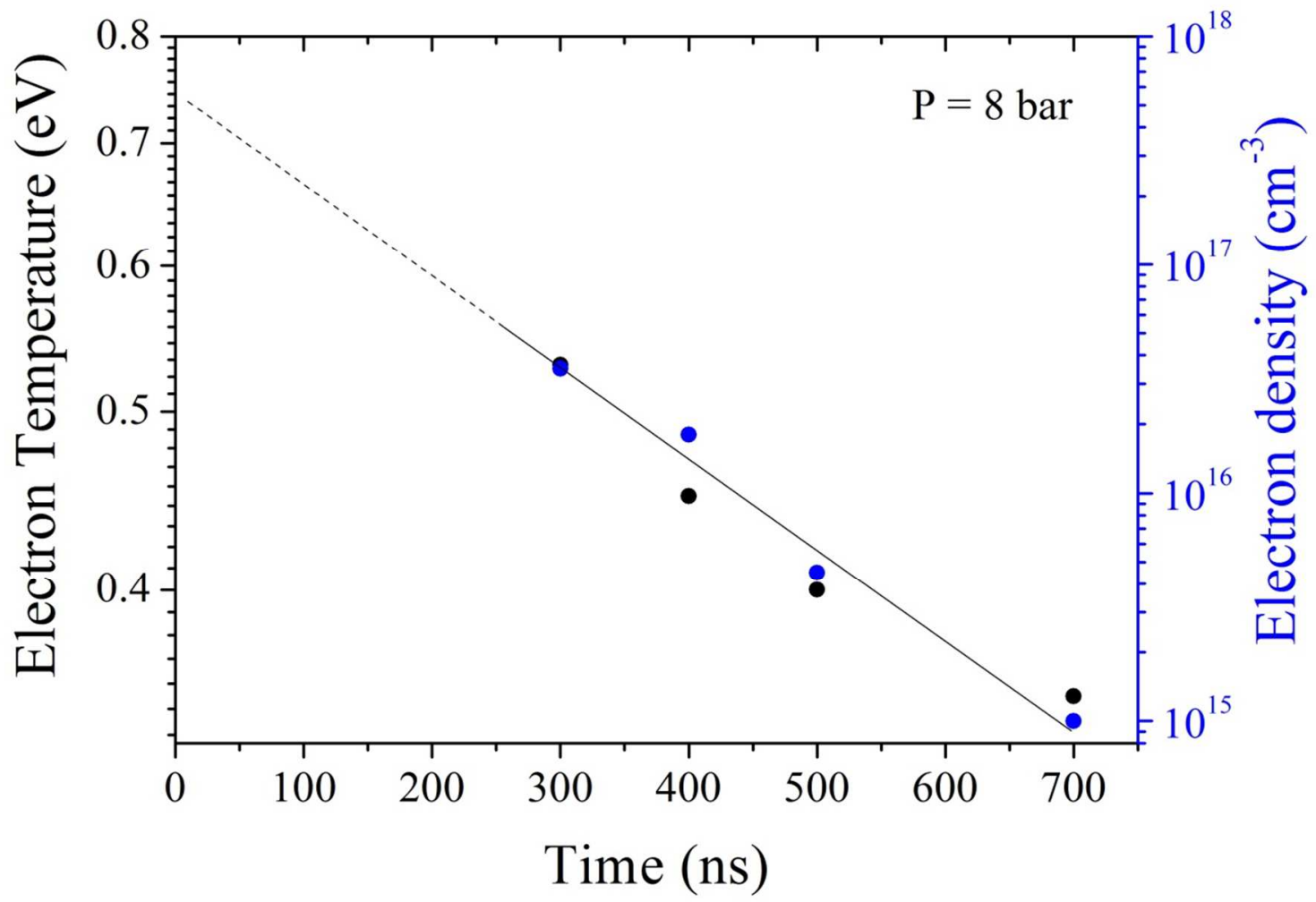

Figure 7: Time-evolution of the electron temperature and electron density determined theoretically. 


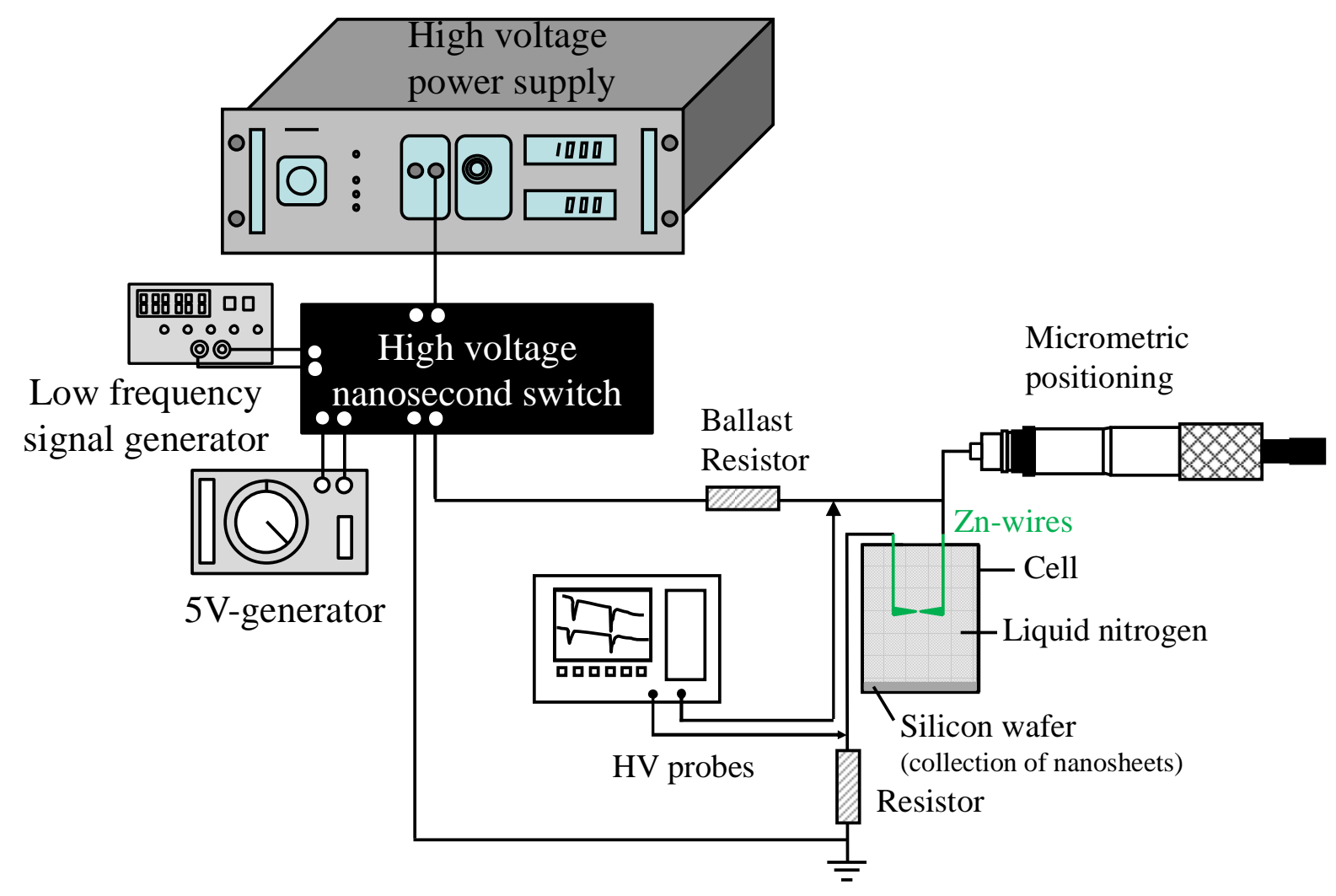

Supplemental Material 1: Schematic of the experimental setup used in this work. 


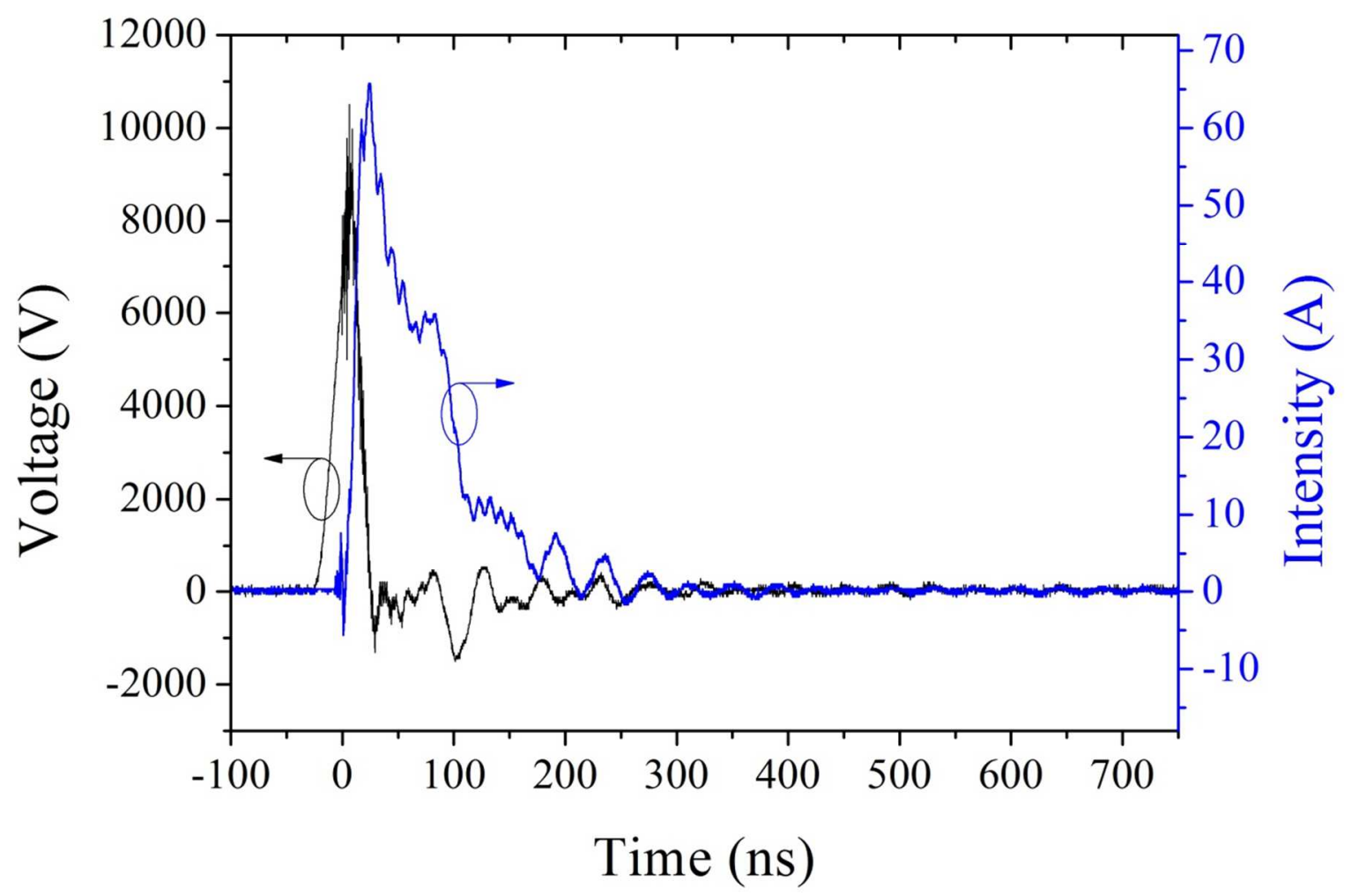

Supplemental Material 2: Voltage and current time-evolutions. Discharge in liquid nitrogen between two zinc electrodes in pin-to-pin configuration. Applied voltage: $+10 \mathrm{kV}$. 


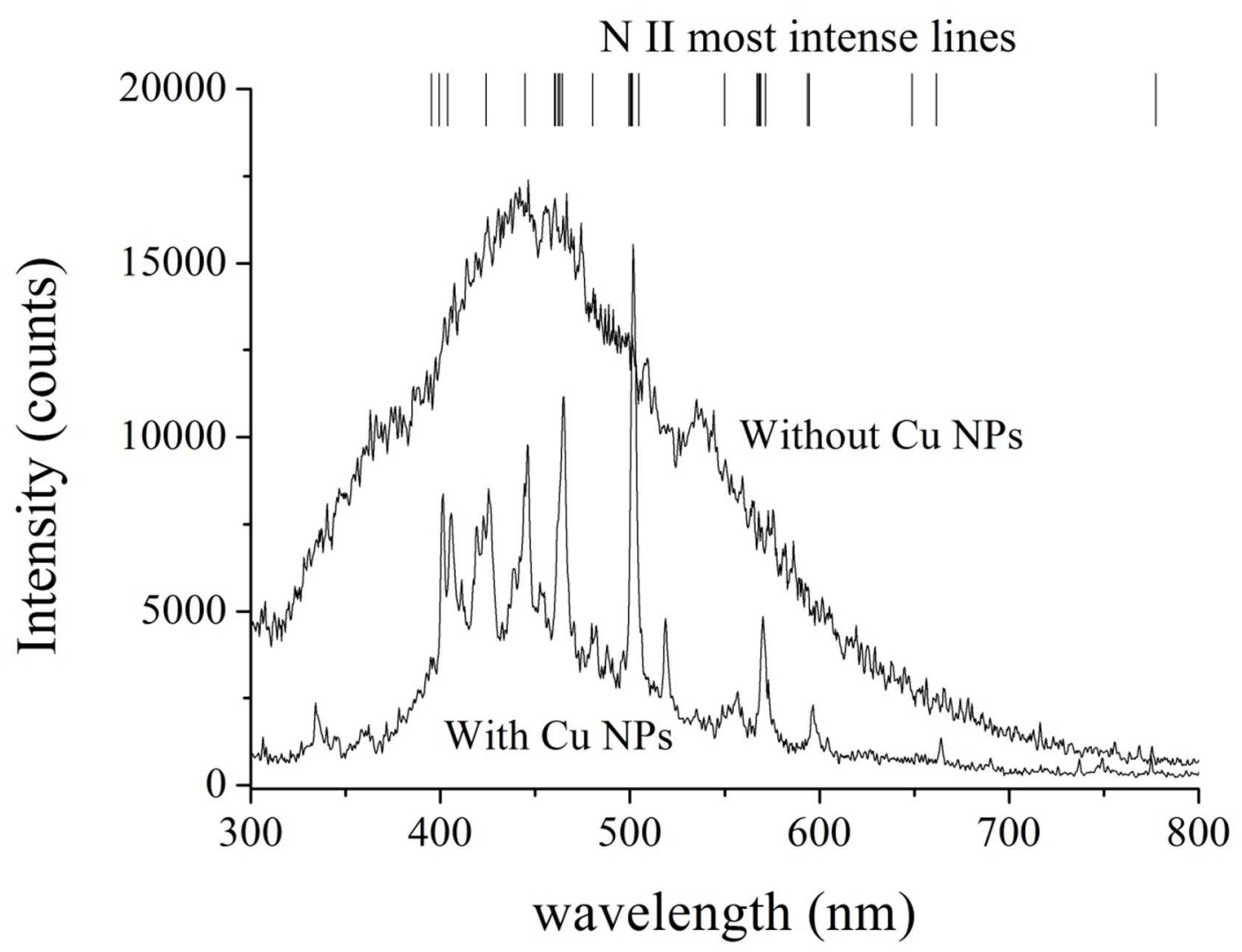

Supplemental Material 3: Intensity emission $100 \mathrm{~ns}$ after breakdown (integration time: 50 ns). Discharge between zinc electrodes in liquid nitrogen with or without copper nanoparticles. Applied voltage: 10 kV. Gap distance: $100 \mu \mathrm{m}$. Pulse duration: 200 ns. Besides $\mathrm{N}$ II lines, only Zn I lines are detected in the time-resolved spectra in both situations (no copper). These data are not corrected from the optical response of the system used to record them. 

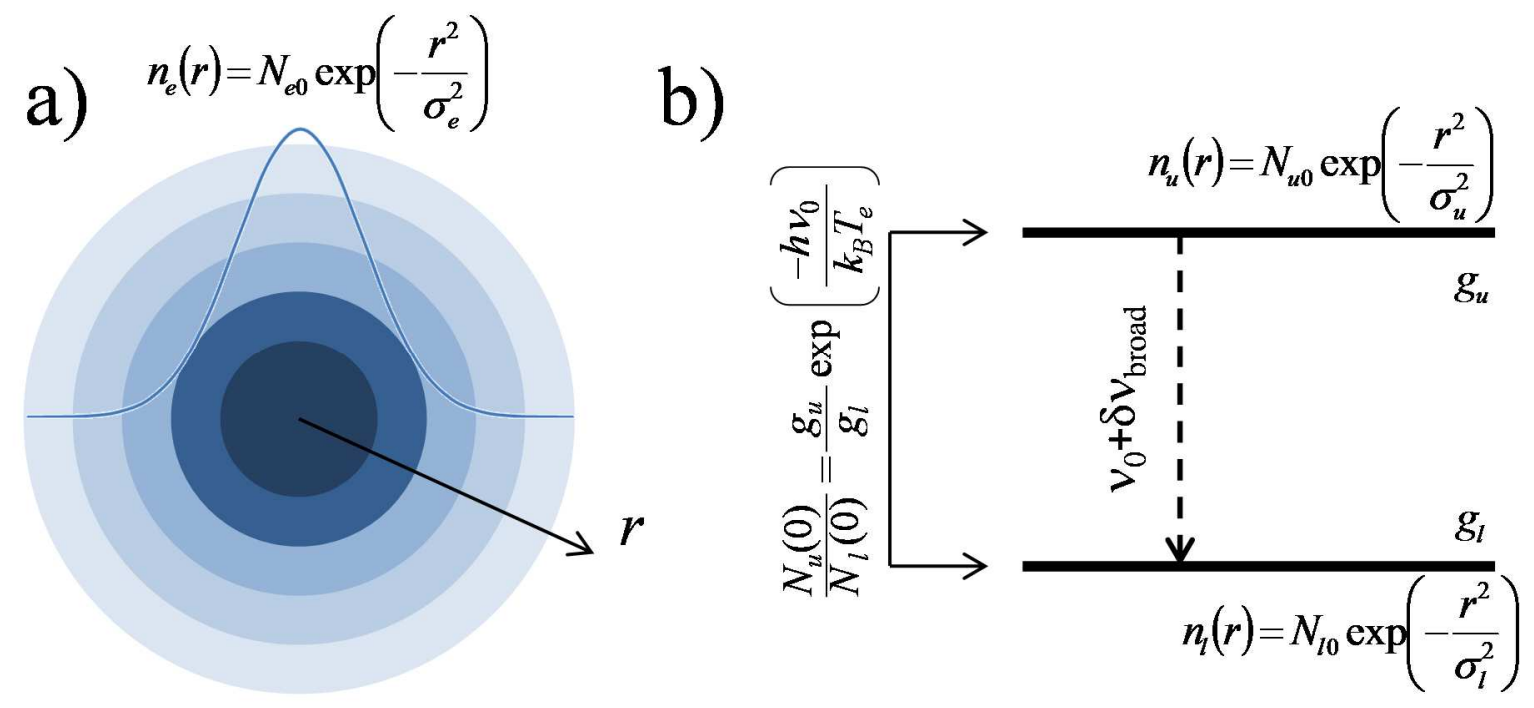

Supplemental Material 4: The optical model used in this work 
Singlet states

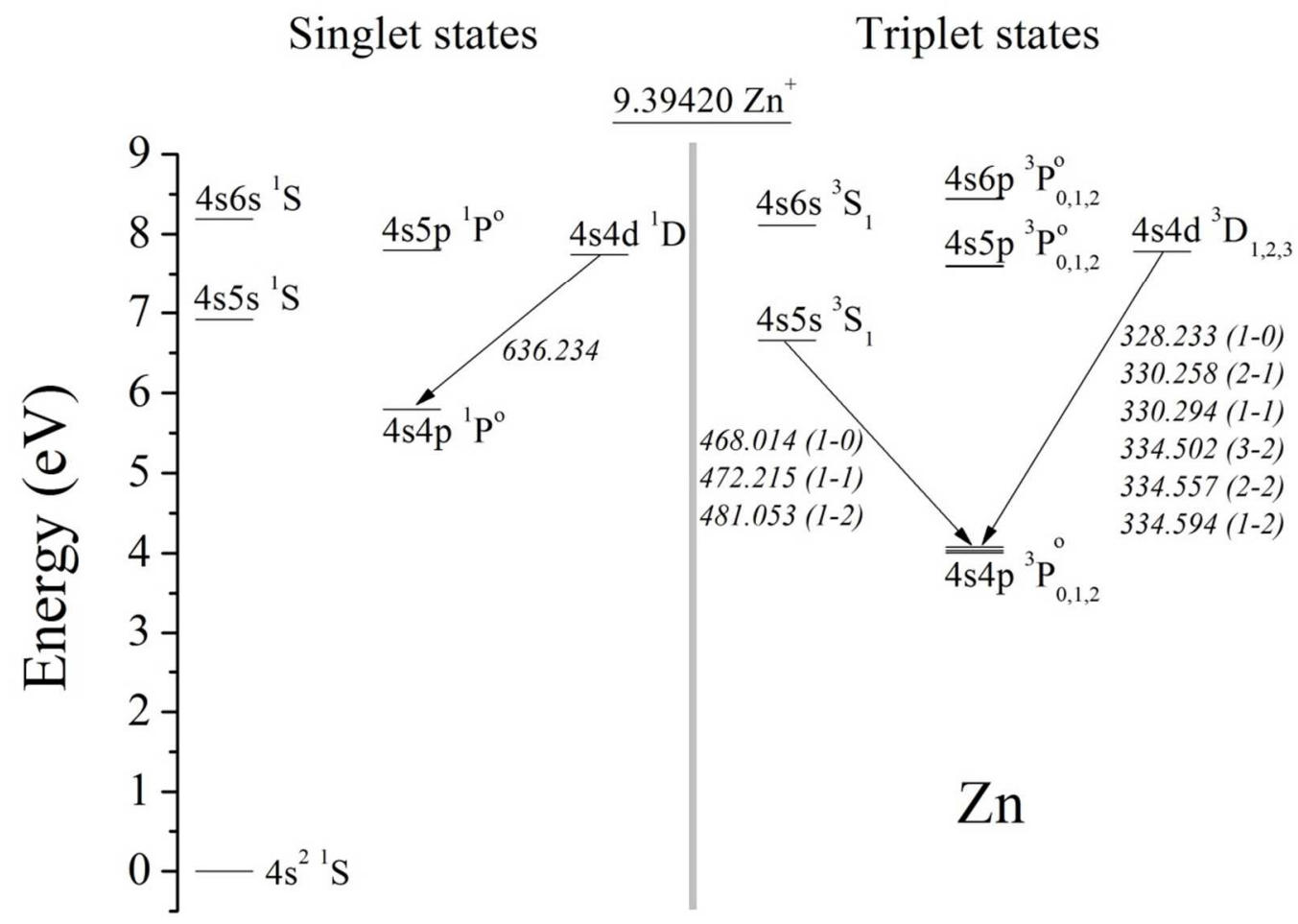

Supplemental Material 5: Grotrian diagram of zinc showing the main transitions observed in this work. 

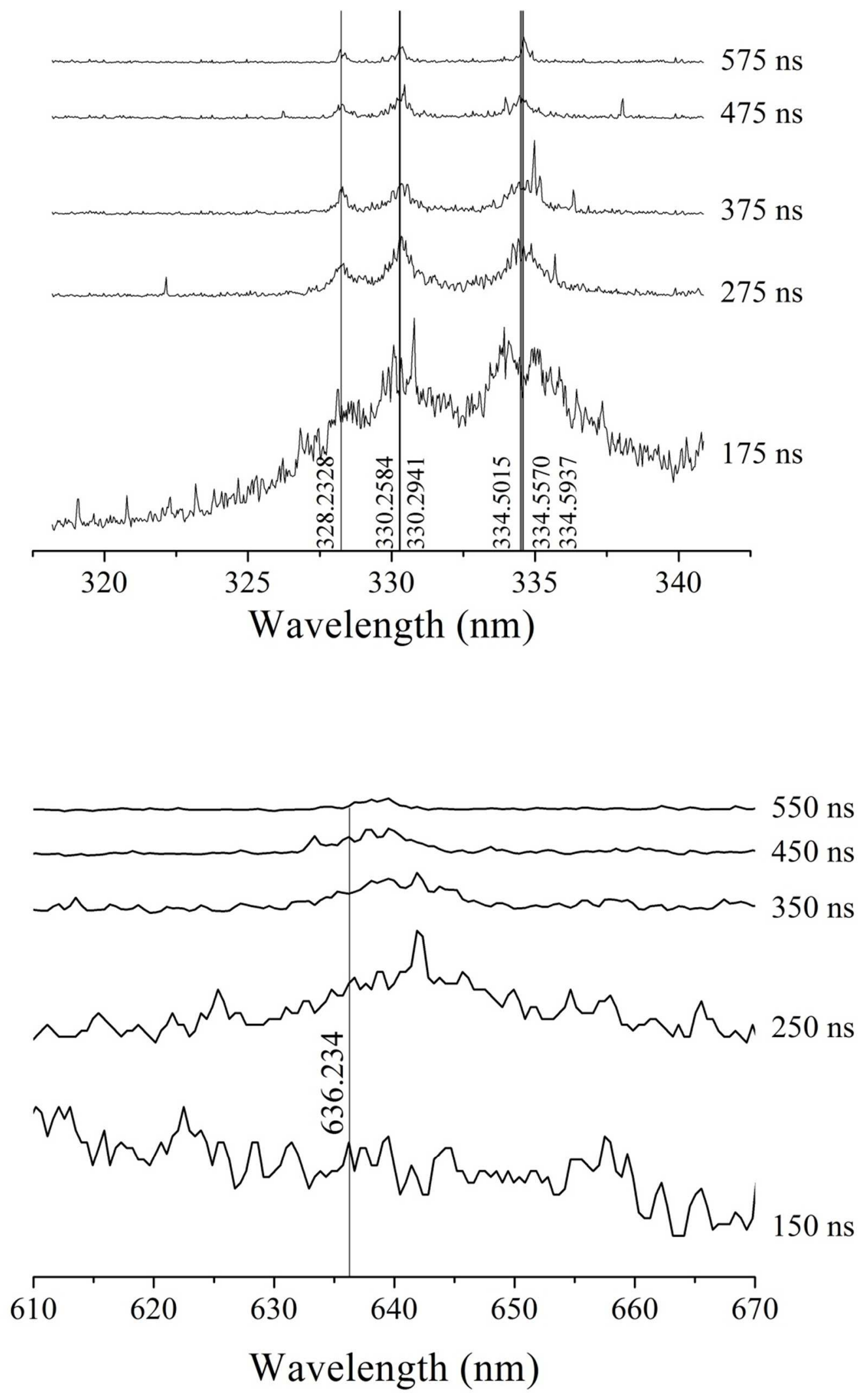

Supplemental Material 6: Example of time-evolution of weak lines. These lines are too noisy to be exploited. 
Table 1: Basic data used in the model and taken from reference [19]

\begin{tabular}{cccccccccc}
\hline $\begin{array}{c}\text { Observed } \\
\text { Wavelength } \\
(\mathrm{nm})\end{array}$ & $\begin{array}{c}\text { Rel. } \\
\text { Int. }\end{array}$ & $\mathrm{A}_{\mathrm{ul}}$ & $\mathrm{E}_{1}$ & $\mathrm{E}_{\mathrm{u}}$ & $\begin{array}{c}\text { Lower } \\
\text { level }\end{array}$ & $\begin{array}{c}\text { Upper } \\
\text { level }\end{array}$ & $\mathrm{C}_{3}^{\prime}$ & $\mathrm{C}_{4}^{\prime}$ & $\mathrm{C}_{6}^{\prime}$ \\
\hline 328.2328 & 500 & $8.6570 \times 10^{7}$ & 4.00609680 & 7.78335510 & 0 & 1 & $3.50 \times 10^{-15}$ & $5.79 \times 10^{-21}$ & $2.00 \times 10^{-41}$ \\
330.2584 & 800 & $1.0720 \times 10^{8}$ & 4.02966260 & 7.78335510 & 1 & 2 & $4.70 \times 10^{-15}$ & $1.09 \times 10^{-20}$ & $1.50 \times 10^{-41}$ \\
330.2941 & 700 & $5.9560 \times 10^{7}$ & 4.02966260 & 7.78273960 & 1 & 1 & $2.00 \times 10^{-15}$ & $1.09 \times 10^{-20}$ & $1.10 \times 10^{-41}$ \\
334.5015 & 800 & $1.5000 \times 10^{8}$ & 4.07788390 & 7.78335510 & 2 & 3 & $5.70 \times 10^{-15}$ & $1.45 \times 10^{-20}$ & $1.00 \times 10^{-41}$ \\
334.5570 & 500 & $3.7490 \times 10^{7}$ & 4.07788390 & 7.78273960 & 2 & 2 & $1.80 \times 10^{-15}$ & $1.45 \times 10^{-20}$ & $1.40 \times 10^{-41}$ \\
334.5937 & 150 & $4.1660 \times 10^{6}$ & 4.07788390 & 7.78233800 & 2 & 1 & 0.00 & $1.45 \times 10^{-20}$ & $1.50 \times 10^{-41}$ \\
468.0134 & 300 & $1.5330 \times 10^{7}$ & 4.00609680 & 6.65451460 & 0 & 1 & $1.00 \times 10^{-15}$ & $1.76 \times 10^{-21}$ & $1.20 \times 10^{-42}$ \\
472.2153 & 400 & $4.5760 \times 10^{7}$ & 4.02966260 & 6.65451460 & 1 & 1 & 0.00 & $4.43 \times 10^{-21}$ & $1.50 \times 10^{-42}$ \\
481.0528 & 400 & $7.0040 \times 10^{7}$ & 4.07788390 & 6.65451460 & 2 & 1 & 0.00 & $4.48 \times 10^{-21}$ & $1.50 \times 10^{-42}$ \\
636.2338 & 1000 & $4.6520 \times 10^{7}$ & 5.79569250 & 7.74387400 & 1 & 2 & $1.40 \times 10^{-14}$ & 0.00 & $8.00 \times 10^{-42}$ \\
\hline
\end{tabular}


Table 2: Parameters of Eq. (27) as determined from experimental data for the three studied zinc lines.

\begin{tabular}{cccc}
\hline Wavelength $(\mathrm{nm})$ & $w_{e}(\mathrm{~nm})$ & $d_{e}(\mathrm{~nm})$ & $a\left(T_{e}\right)$ \\
\hline 468.0134 & 0.28 & 0.10 & 0.25 \\
472.2153 & 0.265 & 0.14 & 0.35 \\
481.0528 & 0.245 & 0.16 & 0.49 \\
\hline
\end{tabular}


Table 3: Contribution of broadening sources expressed in \% at two different times.

\begin{tabular}{|c|c|c|c|c|c|c|c|}
\hline Wavelength (nm) & Instrumental & Doppler & $\begin{array}{l}\text { Pressure } \\
\text { (impact) }\end{array}$ & $\begin{array}{c}\text { Pressure } \\
\text { (Quasi-static) }\end{array}$ & Resonance & Stark & Total (nm) \\
\hline \multicolumn{8}{|c|}{$300 \mathrm{~ns}$} \\
\hline 468.0134 & 2.93 & 0.11 & 0.33 & 0.00 & 2.19 & 94.44 & 2.73 \\
\hline 472.2153 & 2.89 & 0.11 & 0.37 & 0.00 & 0.00 & 96.63 & 2.79 \\
\hline 481.0528 & 2.80 & 0.11 & 0.35 & 0.00 & 0.00 & 96.74 & 2.86 \\
\hline \multicolumn{8}{|c|}{$700 \mathrm{~ns}$} \\
\hline 468.0134 & 32.24 & 0.82 & 4.90 & 0.00 & 35.51 & 26.53 & 0.25 \\
\hline 472.2153 & 49.38 & 1.25 & 8.13 & 0.63 & 0.00 & 40.63 & 0.16 \\
\hline 481.0528 & 49.38 & 1.25 & 8.75 & 0.63 & 0.00 & 40.00 & 0.16 \\
\hline
\end{tabular}

